\title{
Radiative transfer equation and direct simulation prediction of reflection and absorption by particle deposits
}

\author{
Bahareh Ramezanpour, Daniel W. Mackowski* \\ Department of Mechanical Engineering,3418 Wiggins Hall, Auburn University, $A L$ \\ 36849-5341, USA
}

\begin{abstract}
Two methods for computing the normal incidence absorptance and hemispherical reflectance from plane parallel layers of wavelength-sized spherical particles are presented. The first method is based on an exact superposition solution to Maxwell's time harmonic wave equations for a system of randomlypositioned spherical particles excited by an incident plane wave. The second method is based upon the scalar radiative transport equation (RTE) applied to a plane parallel medium. Comparisons are made using five values of particle refractive index, sphere size parameters ranging from 1 to 4 , and particle volume concentrations ranging from 0.05 to 0.4 . The results indicate that the multiple sphere $T$ matrix method (MSTM) and RTE predictions of hemispherical reflectance and absorptance converge when particle volume fraction becomes small. At higher volume fractions the RTE can yield results for hemispherical reflectance that, depending on the particle size and refractive index, significantly depart from the exact predictions. On the other hand, RTE and MSTM predictions of absorptance have a much closer agreement which is largely independent of the sphere optical properties and volume concentration.
\end{abstract}

Keywords: Multiple scattering, T-matrix method, Adding and doubling, Radiative transport theory, Plane parallel media,

\footnotetext{
*Daniel W. Mackowski

Email address: mackodw@auburn.edu (Daniel W. Mackowski)
}

Preprint submitted to Journal of Quantitative Spectroscopy and Radiative Transfer. November 29, 2016

C) 2016. This manuscript version is made available under the Elsevier user license http://www.elsevier.com/open-access/userlicense/1.0/ 
densely packed media

\section{Introduction}

In recent years, remote sensing methods have been recognized as an important and valuable means to detect and identify the physical and chemical characteristics of particulate material deposited on natural or artificial surfaces.

5 Obviously, the interpretation of remote sensing observations depends on an accurate prediction of scattering properties of bulk materials. In principle, such properties are fundamentally governed by the macroscopic Maxwell's wave equations (MWE)s, yet the microscopic-level complexity of particle deposits has, until recently, made direct solution of MWEs for deposits unfeasible. Because of this, effective medium theories and radiative transport models have traditionally been used to estimate the reflectance and absorption properties of deposits [1, 2, 3, 4. In effective medium theories, an inhomogeneous deposit is modeled as an effectively homogeneous layer characterized by an effective refractive index. This quantity can be obtained by an appropriate mixing rule as a function of particle concentration, pair correlation function, and single scattering properties. The coherent reflection and transmission by the layer can then be obtained by applying the Fresnel relations to the effectively homogeneous film [5, 6, 7].

The phenomenological scalar radiative transport (RT) theory treats the object as an absorbing and scattering medium, with an albedo, extinction coefficient, and scattering phase function obtained from the single scattering properties of the constituent particles. Reflection and absorption can be computed from an appropriate solution technique to the RT equation. A comprehensive examination of the relation of RT theory to the fundamental-level, Foldy-Lax description of multiple scattering has been presented recently by Mishchenko et al. 8]. Among the conditions inherent in RT theory are the assumptions that each particle lies in the far-field region of the other particles, and that the relative positions of the particles are uncorrelated. In closely-packed media 
these assumptions are not valid. However, there is a growing progress to modify and apply the RT methodology to densely-packed particulate systems, via dense-media RT theories [9, 10, 11.

In contrast to the techniques mentioned above, a superposition solution to the MWEs can provide, in principle, an exact method to calculate the reflectance and absorptance of particle deposits [12. Such a method can be viewed as a direct simulation: the solution would be applied to a microscopically-detailed configuration of particles and would provide a detailed description of the scattered field resulting from plane wave incidence and the associated reflectance and absorptance of the configuration. Such methods are, by definition, computationally intensive, as the degrees of freedom in the solution will be directly 40 proportional to the number of particles.

This research examines two methods to predict the radiative properties of plane-parallel layers of wavelength-sized spherical particles. The first method is the exact multiple sphere superposition method, coupled with high-performance computational strategies. We use the FORTRAN 90 implementation of this solution, the multiple sphere $T$ Matrix (MSTM) code, to perform direct simulations of EM wave propagation in large-scale systems of spheres [13, 14. This technique is used in order to study the effects of particle concentration on the spectral directional-hemispherical reflectance and directional absorptance [15] in dense media. The second method is the adding and doubling algorithm for solution of the scalar RTE applied to a plane parallel medium [16, 17, combined with Mie theory to compute the extinction coefficient, albedo, and scattering phase function of the spherical particles.

This paper begins by describing the mathematical formulation of the MSTM and RT techniques. In section 3, a comparison is made between these two methods for five values of particle material refractive index. The refractive index values include both large and small magnitudes of the real and imaginary parts, and are representative of highly refractive pigments and gold in the visible wavelengths and mineral matter in the visible and mid IR. The MSTM target models, in these computations, are comprised of randomly distributed spherical 
particles with size parameters $x=\mathrm{k} a=2 \pi a / \lambda=1,1.5,2$, and 4 where $\mathrm{k}, a$ and $\lambda$ denote wavenumber, particle radius and radiation wavelength. Targets are generated with particle volume fractions varying from 0.05 to 0.4 , and the dimensionless target thickness, $\mathrm{k} H$, ranges from a monolayer (i.e., $\mathrm{k} H_{T} \sim 2 x$ ) to values on the order of $50-100$.

As mentioned earlier, the framework of the RT theory is based on the mutual far-field and uncorrelated positions for the particles, and this approximation will fail in a densely packed medium. Therefore, an additional purpose of this work is to test the accuracy of the scalar RTE in predicting the absorption and hemispherically-integrated reflection for media with relatively large particle volume fractions. In addition, the MSTM method becomes impractical for deposits with large optical thickness. In this respect, by using the simulations to identify the errors between the exact and RTE results, as well as classifying these errors based on the sphere refractive index, size parameter, deposit volume fraction, and optical thickness, we hope to identify the conditions under which the fast and efficient RT procedure can be applied to particle deposits.

\section{Computation methods}

\subsection{Multiple sphere T Matrix method}

The MSTM method was used to calculate light scattering and absorption properties of targets consisting of spherical particles. This method implements the analytical solution to the time harmonic MWEs for a cluster of $N_{S}$ spheres, with each sphere characterized by size parameter $x_{i}=\mathrm{k} a_{i}$, complex refractive index $m_{i}=m_{i}^{\prime}+i m_{i}^{\prime \prime}$ and position vector $\mathbf{r}_{i}$, for $i=1,2, \ldots N_{S}$. At points external to the spheres, the vector electric field amplitude will be given as the superposition of the incident field and fields scattered from each sphere,

$$
E_{e x t}=E_{i n c}+E_{s c a}=E_{i n c}+\sum_{i=1}^{N_{S}} E_{s c a, i}
$$


The incident and scattered fields are represented by expansions of regular and outgoing vector spherical wave functions (VSWFs),

$$
\begin{gathered}
E_{i n c}=\sum_{n=1}^{L_{S}^{i}} \sum_{m=-n}^{n} \sum_{p=1}^{2} f_{m n p}^{i} N_{m n p}^{(1)}(r) \\
E_{s c a, i}=\sum_{n=1}^{L_{S}^{i}} \sum_{m=-n}^{n} \sum_{p=1}^{2} a_{m n p}^{i} N_{m n p}^{(3)}\left(r-r_{i}\right)
\end{gathered}
$$

in which $N_{m n p}^{(t)}$ denotes the regular $(t=1)$ and outgoing $(t=3)$ VSWF of azumuthal degree $m$, order $n$, and TM/TE mode $p$. In equations (2) and (3), $f^{i}$ and $a^{i}$ are known incident and unknown scattering coefficients, respectively. The objective of this method is to calculate the scattering coefficients for each sphere and to achieve this goal, continuity equations are applied at the surface of each particle in the cluster. To employ the boundary conditions at the surface of sphere $i$, the scattered fields from all the other particles are transformed, using the VSWF addition theorem, to a regular expansion centered about $i$. Mie theory is then used to relate the exciting and scattered fields for the sphere[18]. This leads to a system of equations for the scattering coefficients,

$$
a_{m n p}^{i}+\bar{a}_{n p}^{i} \sum_{\substack{j=1 \\ j \neq i}}^{N_{s}} \sum_{l=1}^{L_{S}^{i}} \sum_{k=-l}^{l} \sum_{q=1}^{2} H_{m n p k l q}^{i j} a_{k l q}^{j}=\bar{a}_{n p}^{i} f_{m n p}^{i}
$$

where $\bar{a}^{i}$ denotes the Mie scattering coefficient for sphere $i$, which depends on $m_{i}$ and $x_{i}$. The outgoing VSWF translation matrix, $H^{i j}$, depends only on the dimensionless position of $i$ relative to $j$. When the spheres in the cluster have the same truncation limit $L_{S}$, Eq. (4) represents a set of $2 N_{S} L_{S}\left(L_{S}+2\right)$ linear equations for the scattering coefficients. Iterative methods are used to obtain a solution for a given incident plane wave direction and polarization.

The MSTM computations involved clusters (described below) containing on 85 the order of $N_{s} \sim 10^{3}-10^{4}$ spheres. Even on parallel platforms, such large systems of spheres could require days of computational time to obtain a solution to Eq. (4); the main bottleneck being the $N_{s}^{2}$ scaling of the matrix-vector multiplication used to compute the exciting field at each sphere. To make the 
computations feasible a modification of the publicly-available MSTM code was employed. The modification used a discrete Fourier convolution, coupled with the Fast Fourier Transform (FFT) algorithm, to perform the translation operation in Eq. (4). The process involves three basic steps, being 1) translation of the sphere-centered outgoing expansions onto a new set of origins corresponding to a regular lattice, 2) mutual translation among the lattice origins using the FFT convolution, and 3) translation of the regular exciting field, centered about the lattice origins, to the original sphere origins. This procedure can decrease the computational time by up to a factor of 100 . A paper detailing the procedure is currently in preparation.

Cylindrical targets, of radius $R_{T}$ and thickness $H_{T}$ were created by a simple Monte Carlo scheme which randomly filled the volume with $N_{S}$ spheres of radius $a$; spheres were not allowed to overlap, and a sphere was considered inside the target when the sphere origin was inside the target volume. The volume fraction of the spheres was estimated as

$$
f_{v}=\frac{4 N_{S} a^{3}}{3 R_{T}^{2} H_{T}}
$$

A configuration of spheres, corresponding to a volume fraction of 0.3 , is shown in Fig. 1. To simulate plane-parallel conditions, the target radius $R_{T}$ should be considerably larger than all other length scales present in the problem. For the sphere size parameters used in this investigation (1-4) and most of the volume fraction/target thickness combinations, target radii of $\mathrm{k} R_{T} \sim 40-60$ were sufficient to produce reflectance results that were invariant to increasing $R_{T}$.

For each solution for a target, the scattered power into the backwards hemisphere was obtained by numerical quadrature of the scattered intensity, obtained for two mutually orthogonal linear polarization states of the incident wave, over the hemisphere. The directional-hemispherical reflectance was then obtained ${ }_{110}$ by dividing the integrated power by the target cross section $\pi R_{T}^{2}$. Directional absorptance was calculated from the ratio of the total absorption cross section of the target to the target cross section. The simulation methodology does not 
allow a direct calculation of the directional-hemispherical transmittance of the target, due to the effect of diffraction by the finite-radius target on the forward scattered field. However, for highly absorbing, optically thick targets, for which the transmittance would be expected to be vanishingly small, the results would consistently yield reflectance + absorptance to within $2 \%$ of unity.

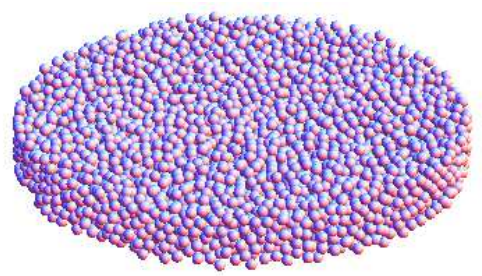

Figure 1: Spherical particle target sample used in MSTM computations.

A difficult situation to model was that corresponding to moderate to high optical thickness and small volume fraction. This could create a target with a dimensionless thickness on the order of $\mathrm{k} H_{T} \sim 100$, and thus require a target radius that was, at least, two or three times this amount. For such conditions the standard (non-FFT based) solution strategy was used in MSTM; due to the relatively large spacings between the spheres in low $f_{v}$ conditions the iterative solution converges very rapidly.

\subsection{Radiative Transfer Theory}

The adding and doubling method (AD) was used to generate numerical solutions to the scalar RTE for plane-parallel media [16, 19, 20. The scalar (or phenomenological) RTE is an integro-differential equation for the specific spectral intensity, and provides an approximation to the specific intensity relative to that obtained from the vector RTE. For the case of directional-hemispherical reflectance and transmittance, the error in this approximation is expected to be on the order of a few percent [21]. Mie theory was used to calculate the extinction cross section, single-scattering albedo, and scattering phase functions of the particles. Adding-doubling is a recursive method, in which the bidirectional reflection and transmission matrices for a combined pair of layers are generated 
from the scattering properties of the individual layers. Initial values of the reflection and transmission matrices are obtained from the optically-thin limit of the RTE, for which the matrices become functions solely of the single scattering properties of the particles. The numerical computation is implemented by means of the Radau quadrature formula in the zenith direction and employing equally spaced points for azimuth dependency. The required number of quadrature points, for a specific set of sphere properties, was established empirically. The calculations were validated against the results of the vector radiative transport equation for the case of Rayleigh scattering [22]. In addition, the results for all sphere classes satisfied energy conservation to within a fraction of a percent.

\section{Computational results}

The MSTM calculations presented in this work were run on the Auburn University College of Engineering compute cluster, using 16 processors. Each MSTM data point corresponds to the average generated from 10 randomlysampled configurations of the target. The amount of time required to generate a point could vary from 10 minutes to 3 days, depending on the number of spheres in the configuration and the sphere size parameter and refractive index. The adding and doubling code was written in MATLAB language and run on a desktop computer. Given the scattering phase function, as calculated by standard Lorenz-Mie theory, the computational time to calculate reflection and transmission matrices over the presented range of optical thicknesses was typically around 2 hours.

Shown in Figs. 26 are predictions of hemispherical reflection versus optical depth as calculated by RT and MSTM procedures for refractive index of $m=$ $2.5,1.54,1.9+0.03 i, 1.46+2.75 i$, and $0.15+3.15 i$. The first, second, and last values are typical of pigment materials (i.e., $\mathrm{TiO}_{2}$ ), quartz/fused silica, and gold in the visible wavelengths, whereas the third and fourth can be associated with quartz at certain IR wavelengths. The plots $(a, b, c$, and $d)$ within each figure correspond to a fixed value of size parameter and curves within each plot show 
the MSTM results for various volume fractions and the RT estimation. The incidence is normal for all calculations, and reflectance is plotted vs. optical thickness $\tau$, which is defined by

$$
\tau=\frac{3 f_{v} H_{T} Q_{e x t}}{4 a}
$$

where $Q_{\text {ext }}$ is the Mie extinction efficiency for the sphere. For a fixed sphere size parameter and refractive index, the RT hemispherical reflectance is a function solely of $\tau$, whereas the MSTM reflectance (for a sufficiently large target radius $\mathrm{k} R_{T}$ ) will depend on the individual values of $\mathrm{k} H_{T}$ and $f_{v}$. Note that all of the MSTM calculations in Figs. 22 6 do not extend to the maximum plotted optical thickness of 4 ; the limit in such cases represents the maximum computationally feasible result for the associated $N_{S}$ and/or target volume.

Several observations can be made regarding the results in these figures. First of all, the predictions of the hemispherical reflection by the RTE and MSTM converge when the particle volume fraction in the medium is less than around five percent. However, at higher volume fractions the RT results can depart significantly from the exact simulations. Another important observation is that, for a set sphere size parameter and refractive index, the RT reflectance is either consistently larger or smaller than the MSTM reflectance for all examined volume fractions and optical thicknesses. In other words, as $f_{v} \rightarrow 0$, the MSTM results, for fixed sphere properties, monotonically approach the RT limit from either above or below. It is worth emphasizing that we were unable to associate the sign or magnitude of the MSTM-RTE difference with the single scattering efficiency, albedo, or asymmetry parameter of the sphere.

The presented results in the plots can be relevant for the interpretation of the reflection behavior of densely packed media based on the size parameters of particles. One can see from Figs. 244 that, for refractive indices with a small (or zero) imaginary part $m^{\prime \prime}$, the differences between RT and MSTM reflectance are greatest for the size parameter of unity and decrease as size parameter increases. However, the situation is reversed for refractive indices with a large imaginary part. As the results in Figs. 5 and 6 demonstrate, the greatest differences 


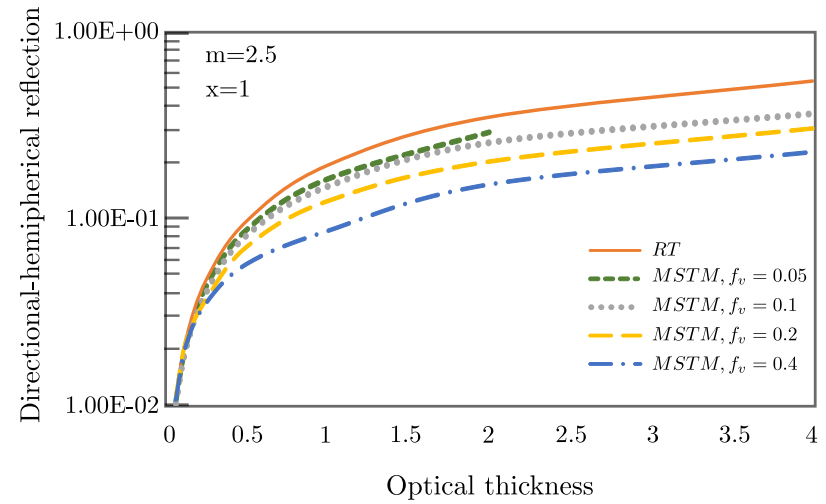

(a)

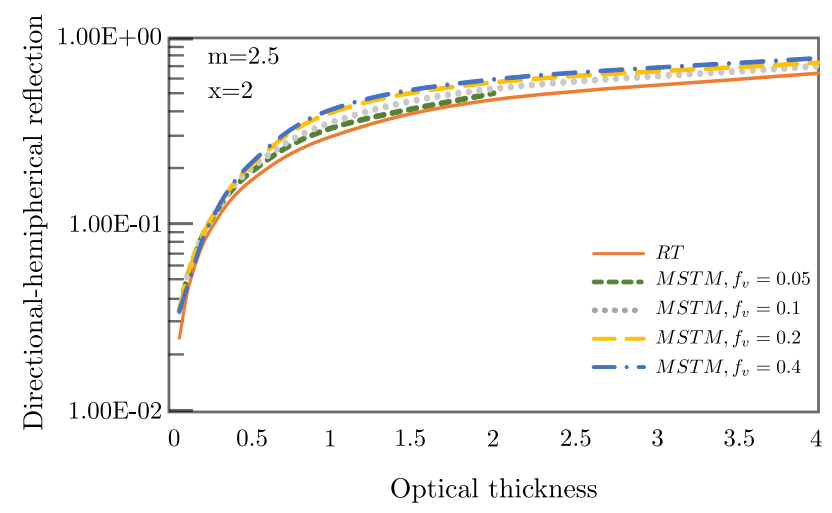

(c)

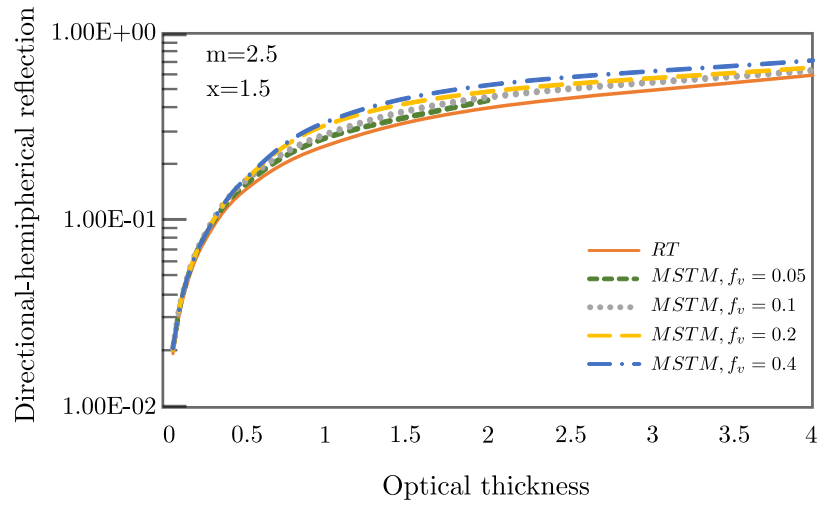

(b)

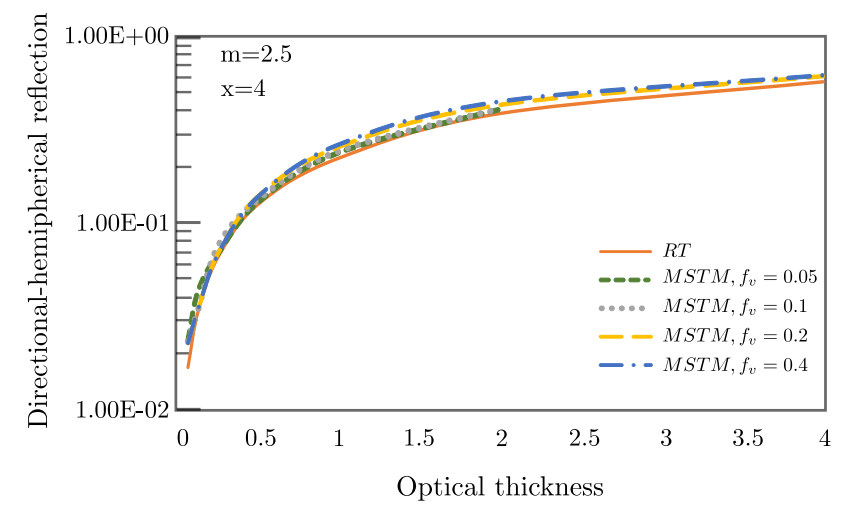

$(d)$

Figure 2: MSTM and RT computations for directional-hemispherical reflection from the modeled deposit with refractive index of $m=2.5$ as a function of optical thickness.

between the RT and MSTM reflectance now occur for a size parameter of 4 . In particular, the RT/MSTM differences are nearly negligible for gold particles when $x=1$, yet become appreciable at $x=4$. An additional feature of the large $m^{\prime \prime}$ results is that an increase in $f_{v}$ leads to an increase in reflectance for all particle size parameters. The situation for small or zero $m^{\prime \prime}$, on the other hand, is more complicated: different size parameters can lead to MSTM reflectance 190 either larger or smaller than RT.

The effect of particle concentration on the normal absorptance of the de- 
posit is examined in Figs. 7, 8, and 9, which correspond to refractive indices of $m=1.9+0.03 i, 1.46+2.75 i$, and $0.15+3.15 i$, respectively. In stark contrast to reflectance, there are no significant differences in the MSTM and RT absorptances for the range of volume fractions and size parameters. To offer an interpretation of this behavior, we note again that the RT model is based on two key assumptions: the particles are in their mutual far field, and the particle positions are uncorrelated. This first assumption addresses the field exciting the individual particles, and whether or not it can be represented as a transverse wave. The second, on the other hand, relates to the far-field interference of waves scattered by separate particles, and whether or not such interference will contribute to the scattered power from the system. In this respect, differences between the RT and MSTM predictions of absorptance would be associated primarily with the first mechanism, i.e., near-field effects. Since no significant differences in absorptance are observed, we can conclude that for the conditions examined here - the differences in RT and MSTM reflectance with increasing $f_{v}$ are primarily the result of increasing correlation among the particles. This conclusion is consistent with recent observations by Mishchenko et al., in which an improved correspondence between vector RTE predictions and polarimetric measurements of bidirectional reflection from dense particle systems was obtained by using a modified phase matrix, which takes into account the particle pair correlation function as a function of particle volume fraction 23 .

\section{Conclusions}

The main conclusion of this work - being that the scalar, independentscattering RTE cannot reliably predict the spectral hemispherical reflectance of densely packed systems of wavelength-sized particles - is not especially surprising given the fundamental assumptions inherent in the RTE. A more noteworthy conclusion is that the RTE predictions, when applied to such systems, can be 
the so-called mechanism of dependent scattering - which corresponds simply to conditions under which the RTE is not valid - can either enhance or attenuate reflection from the particle layer. Our limited calculations suggest that as expected - the magnitude of this effect is dependent on the particle volume fraction, yet the direction of the effect (enhancement or attenuation) is an interdependent function of the particle size parameter and refractive index. In particular, we did not observe a correlation between the direction of the dependent scattering effect and a simple single-scattering property of the sphere.

An additional point we wish to make is that the MSTM-based direct simulation of reflection from particle deposits is entirely feasible on relatively modest (by today's standards) parallel computational platforms. As such platforms advance and become more accessible, we expect that direct simulation methodology will emerge as a practical means of calculating reflection and absorption by discretely inhomogeneous layers such as pigment films, regolith, powder coatings, and so on. The finite, 3-D target methodology used in this investigation has shortcomings when applied to optically thick conditions, as such conditions will require targets with large lateral width in order to model plane parallel conditions. We are currently exploring two approaches to extend the direct simulations to optically thick conditions. One approach is to use direct simulation to calculate the reflection and transmission matrices of a target that is optically thin yet contains sufficient particles to account for dependent scattering effects. The adding-doubling method can then be applied, using these matrices, to predict the reflection and transmission from media of arbitrarily larger thicknesses. The second approach is to reformulate the superposition solution to a plane layer by the use of periodic conditions. In addition, we are currently extending the computations to examine polarized bidirectional reflection from particle layers, and the results of these computations will be presented in a subsequent report. 


\section{Acknowledgments}

250

Tom Kulp, and Thomas Reichardt. This work was supported by DOE-NNSA 
[1] S. Chandrasekhar, Radiative transfer, Courier Corporation, 2013.

[2] K.-N. Liou, An introduction to atmospheric radiation, Vol. 84, Academic press, 2002.

[3] C. F. Bohren, Applicability of effective-medium theories to problems of scattering and absorption by nonhomogeneous atmospheric particles, Journal of the atmospheric sciences 43 (5) (1986) 468-475.

[4] M. I. Mishchenko, Z. M. Dlugach, N. T. Zakharova, Direct demonstration of the concept of unrestricted effective-medium approximation, Optics letters 39 (13) (2014) 3935-3938.

[5] V. Bringi, V. Varadan, V. Varadan, Coherent wave attenuation by a random distribution of particles, Radio Science 17 (5) (1982) 946-952.

[6] P. Waterman, N. Pedersen, Electromagnetic scattering by periodic arrays of particles, Journal of Applied Physics 59 (8) (1986) 2609-2618.

[7] V. V. Varadan, A. Lakhtakia, V. K. Varadan, Scattering by threedimensional anisotropic scatterers, IEEE Transactions on Antennas and Propagation 37 (6) (1989) 800-802.

[8] M. I. Mishchenko, J. M. Dlugach, M. A. Yurkin, L. Bi, B. Cairns, L. Liu, R. L. Panetta, L. D. Travis, P. Yang, N. T. Zakharova, First-principles modeling of electromagnetic scattering by discrete and discretely heterogeneous random media, Physics Reports 632 (2016) 1-75.

[9] J. Randrianalisoa, D. Baillis, Radiative properties of densely packed spheres in semitransparent media: A new geometric optics approach, Journal of Quantitative Spectroscopy and Radiative Transfer 111 (10) (2010) 13721388.

[10] L. Tsang, C.-T. Chen, A. T. Chang, J. Guo, K.-H. Ding, Dense media radiative transfer theory based on quasicrystalline approximation with applications to passive microwave remote sensing of snow, Radio Science 35 (3) (2000) 731-749. 
[11] V. P. Tishkovets, K. Jockers, Multiple scattering of light by densely packed random media of spherical particles: Dense media vector radiative transfer equation, Journal of Quantitative Spectroscopy and Radiative Transfer 101 (1) (2006) 54-72.

[12] D. W. Mackowski, M. I. Mishchenko, Calculation of the T-matrix and the scattering matrix for ensembles of spheres, Journal of the Optical Society of America A 13 (11) (1996) 2266-2278.

[13] D. Mackowski, M. Mishchenko, A multiple sphere T-matrix fortran code for use on parallel computer clusters, Journal of Quantitative Spectroscopy and Radiative Transfer 112 (13) (2011) 2182-2192.

[14] D. W. Mackowski, M. I. Mishchenko, Direct simulation of multiple scattering by discrete random media illuminated by gaussian beams, Physical Review A 83 (1) (2011) 013804.

[15] J. R. Howell, M. P. Menguc, R. Siegel, Thermal radiation heat transfer, CRC press, 2010.

[16] J. De Haan, P. Bosma, J. Hovenier, The adding method for multiple scattering calculations of polarized light, Astronomy and Astrophysics 183 (1987) 371-391.

[17] J. E. Hansen, Multiple scattering of polarized light in planetary atmospheres. part i. the doubling method, Journal of the Atmospheric Sciences 28 (1) (1971) 120-125.

[18] D. W. Mackowski, Calculation of total cross sections of multiple-sphere clusters, Journal of the Optical Society of America A 11 (11) (1994) 28512861.

[19] J. Hovenier, Multiple scattering of polarized light in planetary atmospheres, Astronomy and Astrophysics 13 (1971) 7. 
[20] J. W. Hovenier, C. V. van der Mee, H. Domke, Transfer of polarized light in planetary atmospheres: basic concepts and practical methods, Vol. 318, Springer Science \& Business Media, 2014.

[21] A. Lacis, J. Chowdhary, B. Cairns, M. Mishchenko, Modeling errors in diffuse-sky radiation, Geophys. Res. Lett 25 (1998) 135-138.

[22] K. Coulson, J. Dave, Z. Sekera, I960: Tables related to radiation emerging from a planetary atmosphere with rayleigh scattering.

[23] M. I. Mishchenko, D. H. Goldstein, J. Chowdhary, A. Lompado, Radiative transfer theory verified by controlled laboratory experiments, Optics Letters 38 (18) (2013) 3522-3525. 


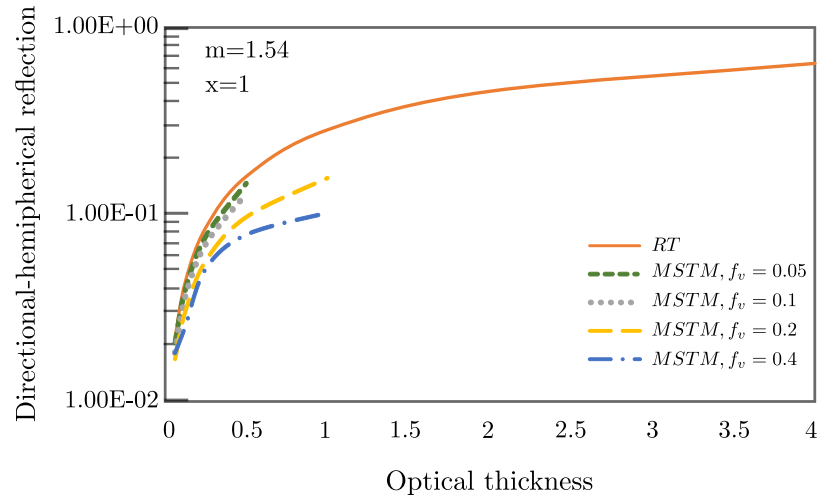

(a)

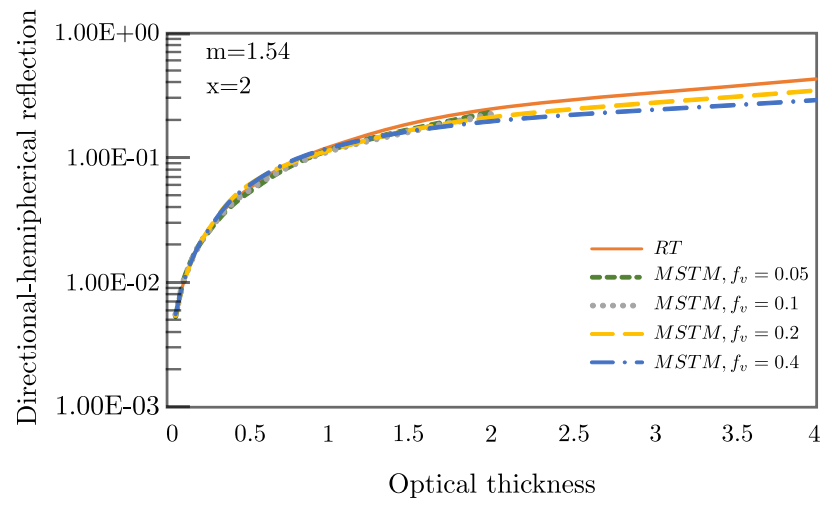

(b)

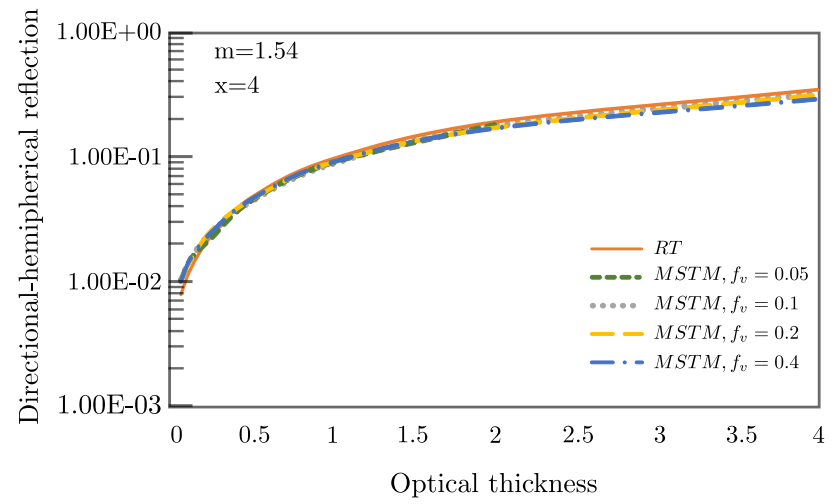

(c)

Figure 3: The same as in Fig. 2 except for the material with $m=1.54$. 


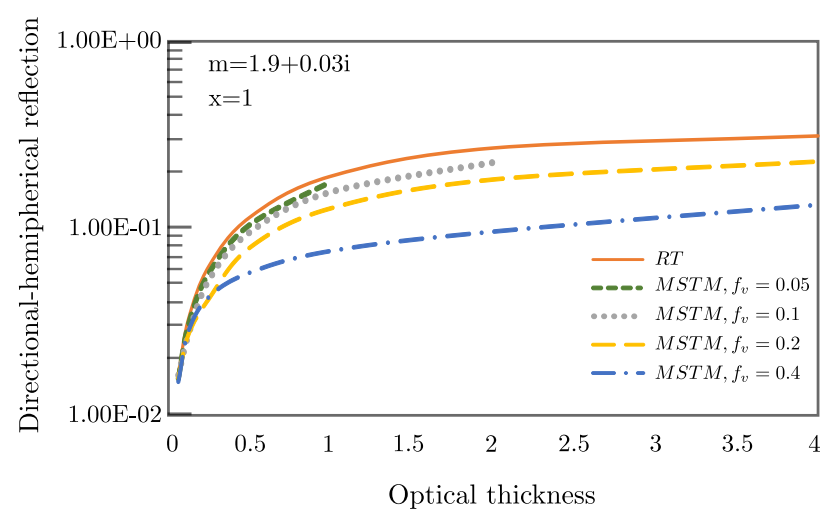

(a)

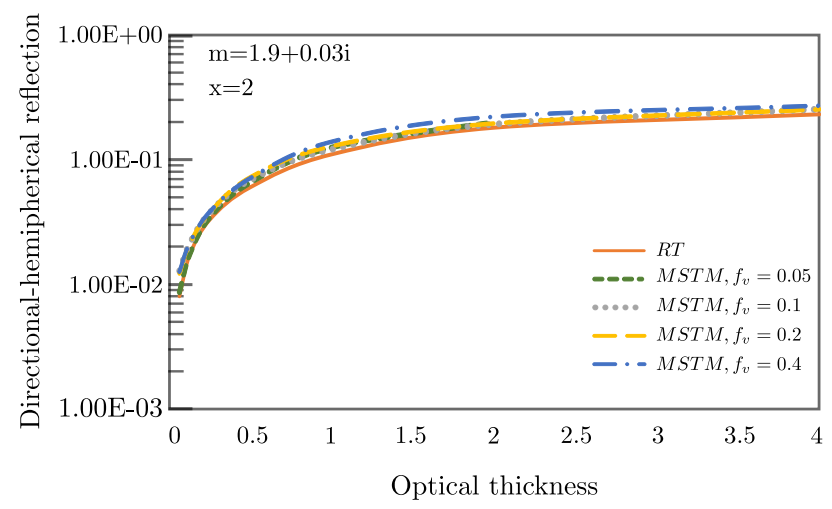

(c)

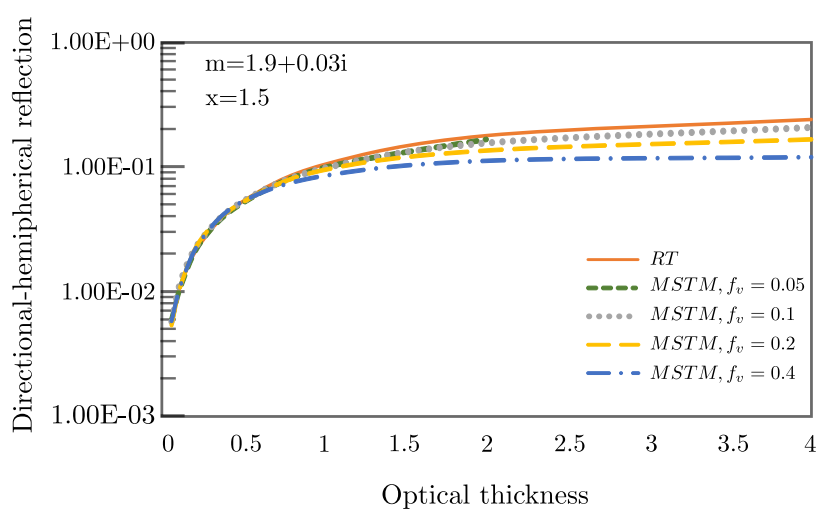

(b)

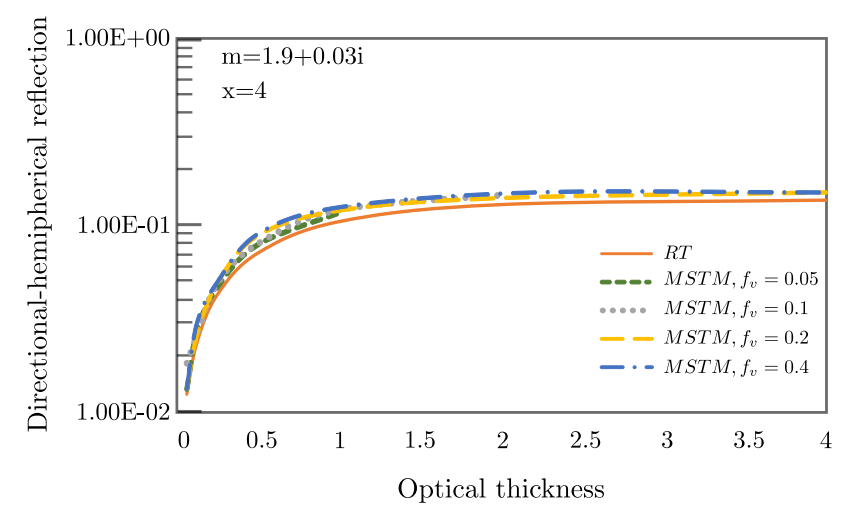

$(d)$

Figure 4: The same as in Fig. 2 except for the material with $m=1.9+0.03 i$. 


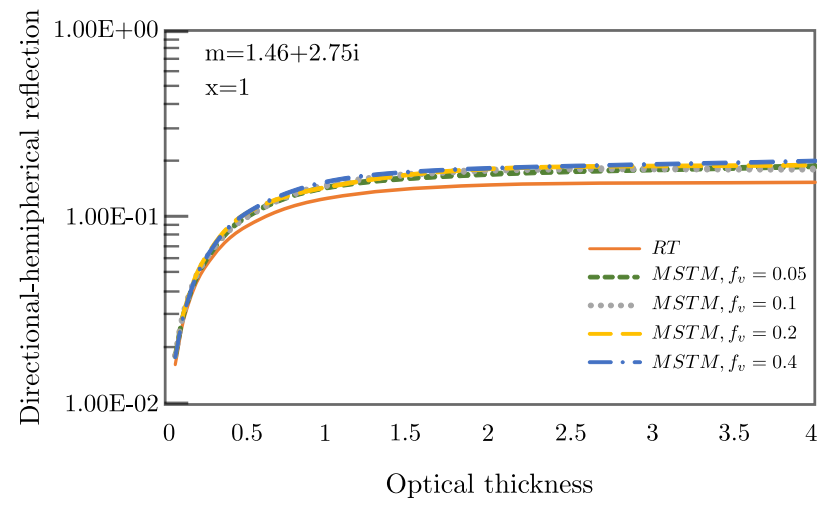

(a)

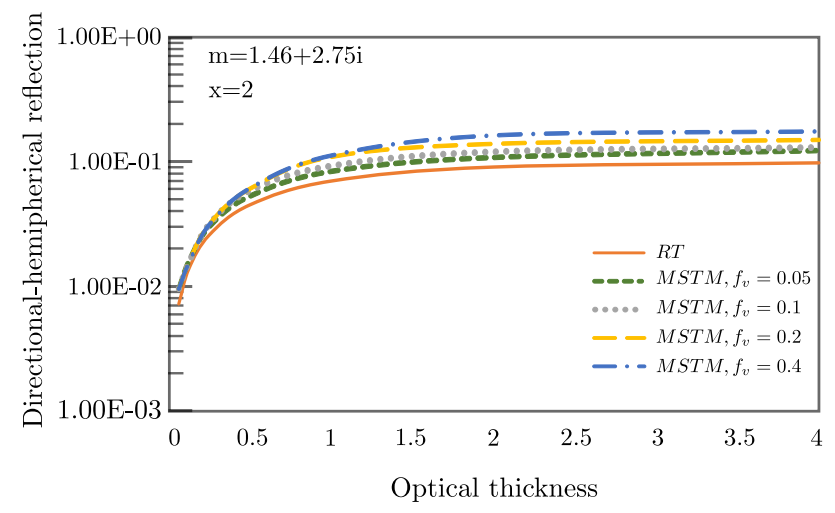

(b)

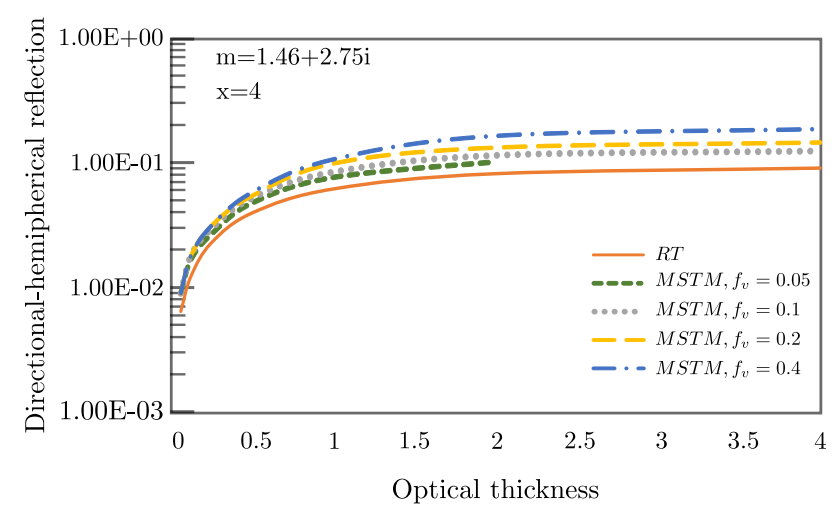

(c)

Figure 5: The same as in Fig. 2 except for the material with $m=1.46+2.75 i$. 


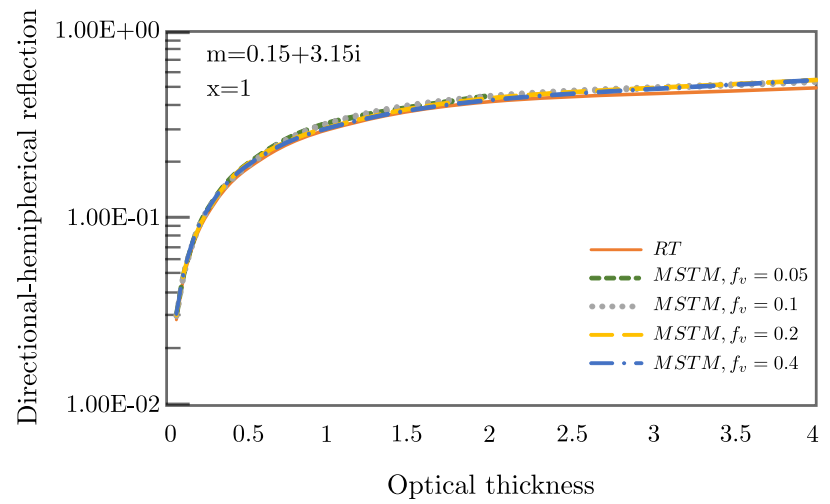

(a)

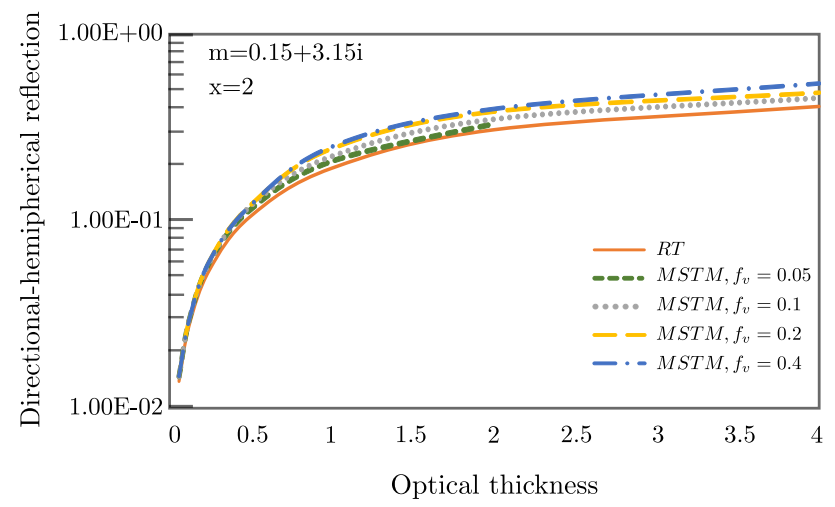

(b)

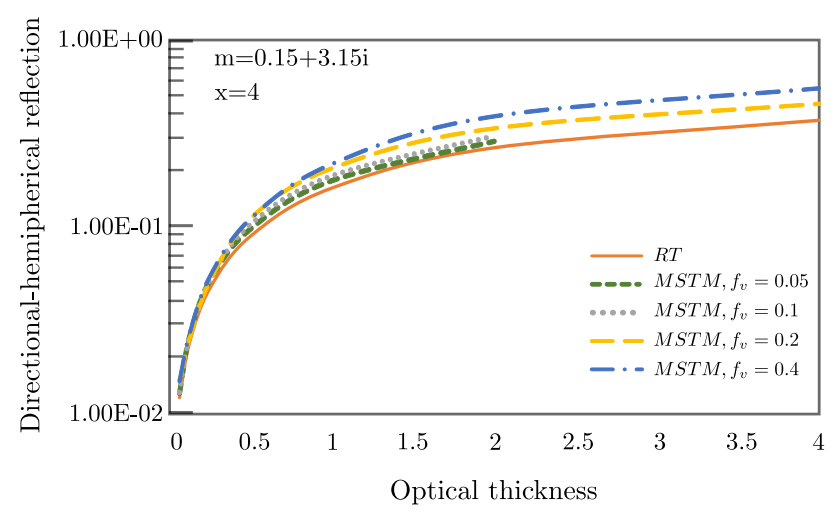

(c)

Figure 6: The same as in Fig. 2 except for the material with $m=0.15+3.15 i$. 


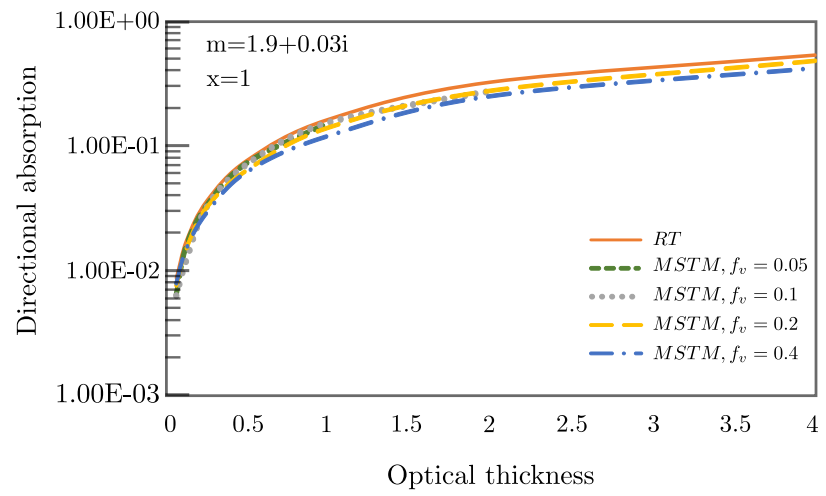

(a)

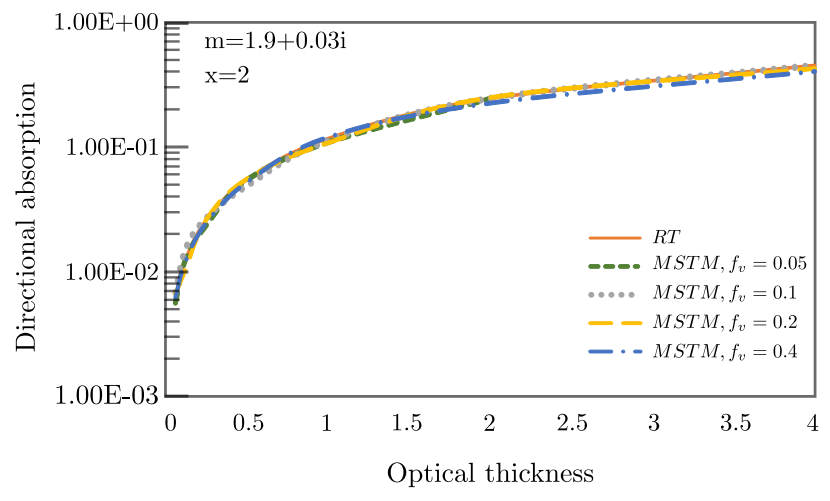

(c)

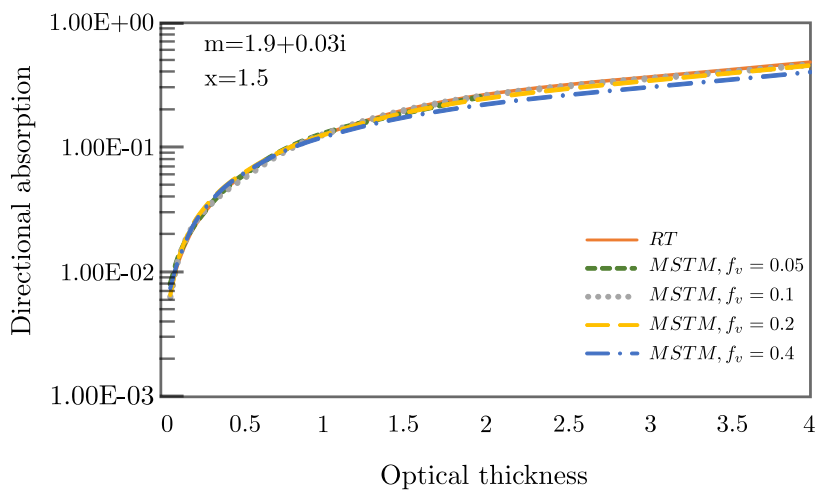

(b)

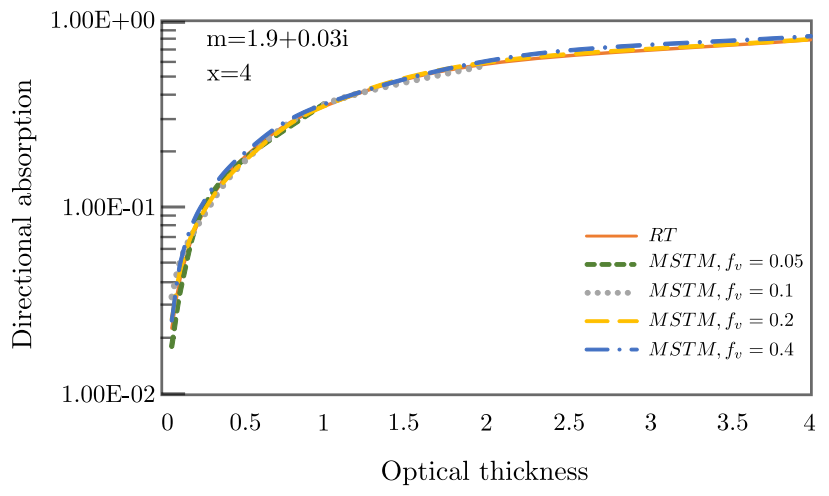

$(d)$

Figure 7: MSTM and RT computations for directional absorption from the modeled deposit with refractive index of $m=1.9+0.03 i$ as a function of optical thickness. 


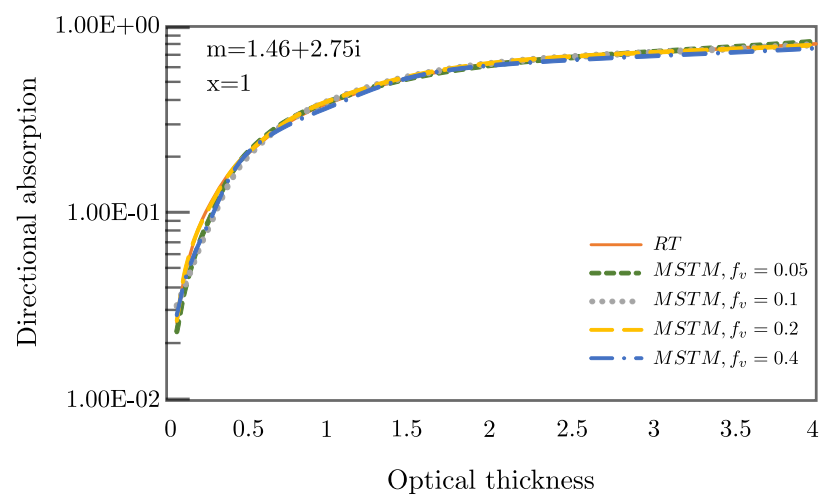

(a)

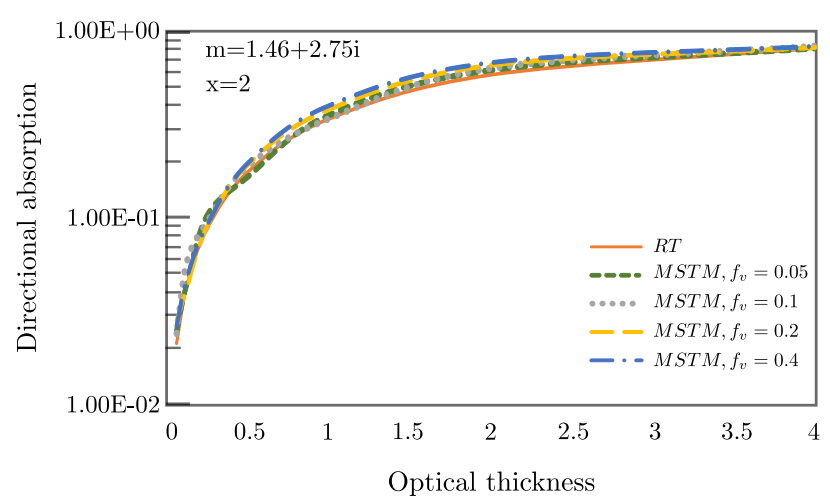

(b)

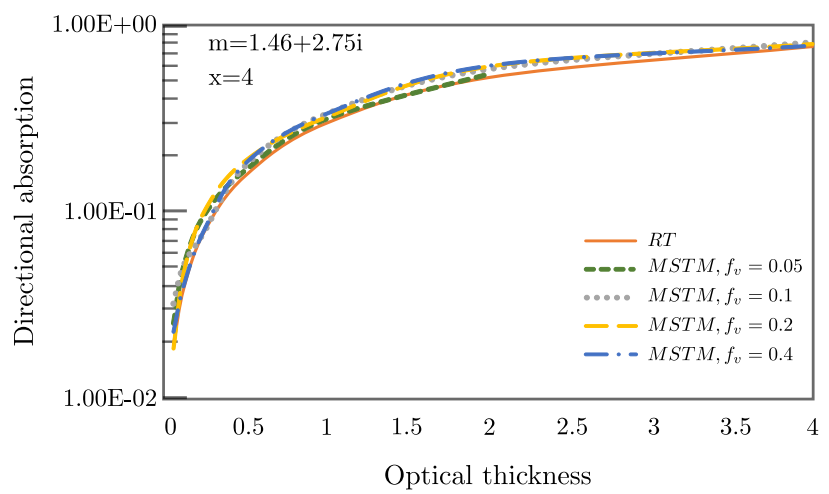

(c)

Figure 8: The same as in Fig. 7 except for the material with $m=1.46+2.75 i$. 


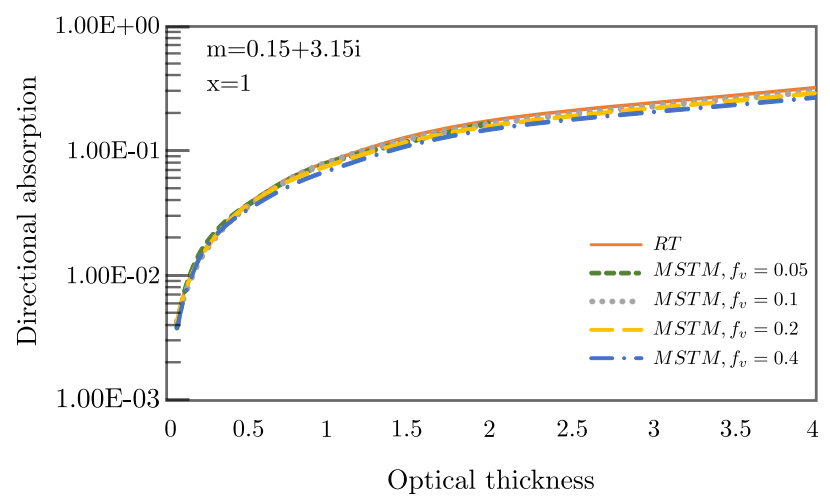

(a)

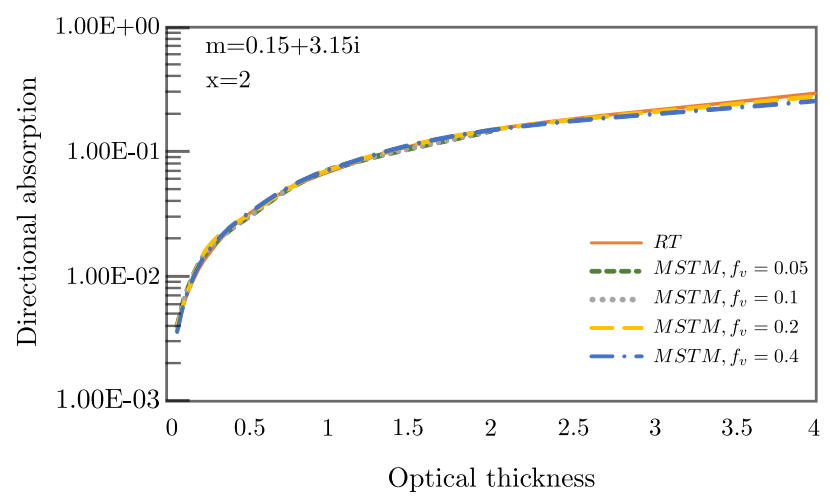

(b)

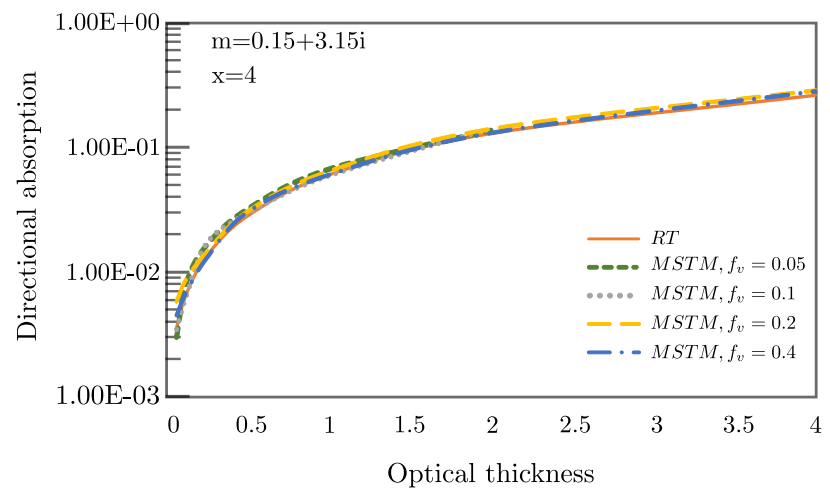

(c)

Figure 9: The same as in Fig. 7 except for the material with $m=0.15+3.15 i$. 